\title{
Rational Design of Thermosensitive Hydrogel to Deliver Nanocrystals with Intranasal Administration for Brain Targeting in Parkinson's Disease
}

\author{
Yun Tan $\left(\mathbb{D},{ }^{1}\right.$ Yao Liu, ${ }^{2}$ Yujing Liu, ${ }^{2}$ Rui Ma, ${ }^{2}$ Jingshan Luo, ${ }^{2}$ Huijie Hong, ${ }^{3}$ Xiaojia Chen (D), ${ }^{3}$ \\ Shengpeng Wang $\left(\mathbb{D},{ }^{3}\right.$ Chuntai Liu $\mathbb{D},{ }^{4}$ Yi Zhang $\mathbb{D},{ }^{1}$ and Tongkai Chen $\mathbb{D}^{2}$ \\ ${ }^{1}$ Hunan Provincial Key Laboratory of Micro \& Nano Materials Interface Science, College of Chemistry and Chemical Engineering, \\ Central South University, Changsha 410083, China \\ ${ }^{2}$ Science and Technology Innovation Center, Guangzhou University of Chinese Medicine, Guangzhou 510405, China \\ ${ }^{3}$ State Key Laboratory of Quality Research in Chinese Medicine, Institute of Chinese Medical Sciences, University of Macau, \\ Macau 999078, China \\ ${ }^{4}$ Key Laboratory of Materials Processing and Mold, Ministry of Education, Zhengzhou University, Zhengzhou 450002, China
}

Correspondence should be addressed to Yi Zhang; yzhangcsu@csu.edu.cn and Tongkai Chen; chentongkai@gzucm.edu.cn

Yun Tan and Yao Liu contributed equally to this work.

Received 19 July 2021; Accepted 18 October 2021; Published 19 November 2021

Copyright (c) 2021 Yun Tan et al. Exclusive Licensee Science and Technology Review Publishing House. Distributed under a Creative Commons Attribution License (CC BY 4.0).

\begin{abstract}
Mitochondrial dysfunction is commonly detected in individuals suffering from Parkinson's disease (PD), presenting within the form of excessive reactive oxygen species (ROS) generation as well as energy metabolism. Overcoming this dysfunction within brain tissues is an effective approach to treat $\mathrm{PD}$, while unluckily, the blood-brain barrier (BBB) substantially impedes intracerebral drug delivery. In an effort to improve the delivery of efficacious therapeutic drugs to the brain, a drug delivery platform hydrogel (MAG-NCs@Gel) was designed by complexing magnolol (MAG)-nanocrystals (MAG-NCs) into the noninvasive thermosensitive poly( $N$ isopropylacrylamide) (PNIPAM) with self-gelation. The as-prepared MAG-NCs@Gel exhibited obvious improvements in drug solubility, the duration of residence with the nasal cavity, and the efficiency of brain targeting, respectively. Above all, continuous intranasal MAG-NCs@Gel delivery enabled MAG to cross the BBB and enter dopaminergic neurons, thereby effectively alleviating the symptoms of MPTP-induced PD. Taking advantage of the lower critical solution temperature (LCST) behavior of this delivery platform increases its viscoelasticity in nasal cavity, thus improving the efficiency of MAG-NCs transit across the BBB. As such, MAG-NCs@Gel represented an effective delivery platform capable of normalizing ROS and adenosine triphosphate (ATP) in the mitochondria of dopaminergic neurons, consequently reversing the mitochondrial dysfunction and enhancing the behavioral skills of PD mice without adversely affecting normal tissues.
\end{abstract}

\section{Introduction}

Parkinson's disease (PD) is one of the most severe neurodegenerative diseases which affects approximately $2 \%$ of individuals over the age of 65 , resulting in motor and nonmotor dysfunction because of the degenerating the dopaminergic neurons in the substantia nigra [1-3]. PD incidence among younger individuals has been rising in recent years and therefore represents a major threat to global public health [4]. At present, drug-based therapeutics are the primary tools used to treat $\mathrm{PD}$, with dopamine receptor agonists, monoamine oxidase B inhibitors, and the dopamine precursor levodopa being the main treatments $[5,6]$. These pharmacological agents can slacken disease progression, alleviate symptoms, and thereby improve patient quality of life. However, most of known drugs are incapable of efficiently penetrating the blood-brain barrier (BBB) and cause substantial systemic adverse effects, limiting their clinical utility $[7,8]$.

The intranasal delivery of PD drugs is often considered to be preferable owing to its ability to decrease systemic drug delivery while by passing the selectivity of the BBB to directly transport pharmacological agents into the brain via 
the trigeminal and olfactory systems that link the external environment directly to the central nervous system (CNS) [9-11]. In recent years, natural products, as promising resources with sustainable and low toxicity, have attracted considerable attentions in the treatment of PD by nasal administration [12-14]. In addition, by evading first-pass hepatic metabolism, this approach also reduces the rates of drug degradation. Several nanoparticle-based strategies, such as solid lipid nanoparticles $[15,16]$, nanoemulsions [17], liposomes [18], polymeric micelles [19, 20], and cyclodextrin derived [21] have been used to achieve efficient intranasal drug delivery to date. Nanocrystal- (NC-) based delivery platforms are particularly promising in this therapeutic context, as they consist of small crystals $(<500 \mathrm{~nm})$ stabilized with surfactants or polymeric steric stabilizers $[22,23]$. The NC delivery platforms enable researchers to achieve targeted therapy and efficient treatment and contribute to transform the balance from side effect therapy to a beneficial one [24, 25]. After production, these NCs can be loaded with drugs of interest at high levels without organic solvents and remain highly stable and less toxic than other nanoscale drug delivery platforms. Despite those innovative advances in fabrication, structural characterization, and formation mechanism [26, 27], as well as the tests of in vitro therapeutic efficacy [28], the clinical application of most NCs has been hindered by their poor solubility and strong self-aggregation tendency [29]. To establish a rational NCbased drug carrier system within the nasal cavity still remains a great challenge.

Hydrogel function is treated as ideal drug carriers owing to their ability to overcome a range of limitations [30-33]. In particular, thermosensitive hydrogels can be prepared and loaded with drugs under relatively mild conditions, exhibiting thermally controlled drug loading/unloading behavior when applied to the nasal mucosa in the form of an injectable preparation owing to their shear thinning properties [34-36]. Poly(N-isopropylacrylamide) (PNIPAM) hydrogel is a typical thermosensitive hydrogel with reversible lower critical solution temperature (LCST) behavior owing to its temperaturedependent hydrophilicity and hydrophobicity [37-39]. The LCST of PNIPAM hydrogels is typically approximately $32^{\circ} \mathrm{C}$ [40]. The exposure of these hydrogels to warmer temperatures within the nasal cavity can result in increased hydrogel viscoelasticity, thereby reducing the rate of mucociliary clearance and ensuring gradual sustained drug release. Such a thermosensitive hydrogel delivery system has offered great potential to improve drug potency and to absorb significant amounts of water or biological fluids [41, 42]. Meanwhile, these hydrogels are also both biocompatible and biodegradable, making them ideal tools for clinical application.

Inspired by abovementioned pioneering works and integrating our previous experience $[8,43,44]$, we attempted to employ the hydroxylated biphenol derived from the 'Houpo' herb (Magnolia officinalis) known as magnolol (MAG) as a model drug, as it is commonly studied to treat PD $[45,46]$. To improve the solubility, stability, and bioavailability characteristics of MAG, PVP-K30 was used to stabilize MAGcontaining NCs (MAG-NCs). Further, in order to construct the MAG-NCs@emulsion, the as-developed MAG-NCs were then uniformly dispersed into an emulsion of thermosensitive PNIPAM, which was obtained by in situ free radical polymerization of precursor (Figure 1(a)). Following, as a self-gelation process, it is wonderful that the MAG-NCs@emulsion polymer changed from a fluid to a solid-like state during natural cooling, yielding the transparent polymer network MAG-NCs@Gel (Movie S1).This MAG-NCs@Gel preparation was then intranasally injected into experimental model mice, whereupon it was able to penetrate the BBB to achieve effective drug release, thereby targeting mitochondria within neurons to reduce ROS generation and increase ATP levels as a treatment for PD (Figure 1(b)). The thermosensitive hydrogel will happen in phase transition and exhibit the characteristics of opacity and volume shrinkage, when the temperature is higher than the LCST of the hydrogel $[47,48]$. According to the relationship between the transparency of the hydrogels and the temperature, the LCST of MAG-NCs@Gel and blank gel have been determined at about $\sim 32.2^{\circ} \mathrm{C}$ and $32.4^{\circ} \mathrm{C}$, respectively (Figure S1). Since the body temperature (of murine and rats) is higher than the LCST of MAG-NCs@Gel, the volume of MAGNCs@Gel will shrink to some extent in these animals. In addition, storage modulus $\left(G^{\prime}\right)$ and loss modulus $\left(G^{\prime \prime}\right)$ of MAG-NCs@Gel increased with rising temperature (Figure S2), indicating that MAG-NCs@Gel with slightly contracted volume exhibited better viscoelasticity in the nasal cavity than the external body. Therefore, these rationally designed MAG-NCs@Gel preparations were able to overcome limited MAG solubility within aqueous environments, while simultaneously achieving prolonged MAG residence in the nasal cavity. Importantly, encapsulated MAG-NCs were able to efficiently transit across the $\mathrm{BBB}$, thus improving their cerebral bioavailability and enabling the targeted treatment of PD.

\section{Results}

2.1. Characterization of MAG-NCs and MAG-NCs@Gel. The dynamic light scattering (DLS) was initially used to assess the hydrated particle size of MAG-NCs, which exhibited a mean diameter of $81.57 \pm 1.48 \mathrm{~nm}$ and the mean particle distribution index (PDI) of $0.11 \pm 0.02$ (Figure S3a), and the mean hydrated MAG-NC particle size for storage one month was still 81-84 nm with PDI of 0.1-0.2 (Figure S3b), these indicating that $\mathrm{MAG}-\mathrm{NC}$ remained stable for a month or more. Relative to the sizes of hydrated particles detected via DLS, TEM imaging suggested MAG-NCs to be somewhat smaller about $30 \mathrm{~nm}$ (Figure S3c), because TEM images revealed the true size of the drug NCs without accounting for the PVP-K30 coating or the electric double layer. To further confirm MAG-NC stability, TEM images of MAG-NCs were captured following storage for two weeks, revealing that particles were still well dispersed, neither agglomeration nor dissociation (Figure S3d). Meanwhile, SEM images of freeze-dried MAG-NCs@Gel indicated the uniform distribution of MAG-NCs within honeycomb polymer network, and the size of MAG-NCs was about 50-70 nm (Figure 1(a)). The SEM of the blank hydrogel shows the homogeneous polymer network, except 


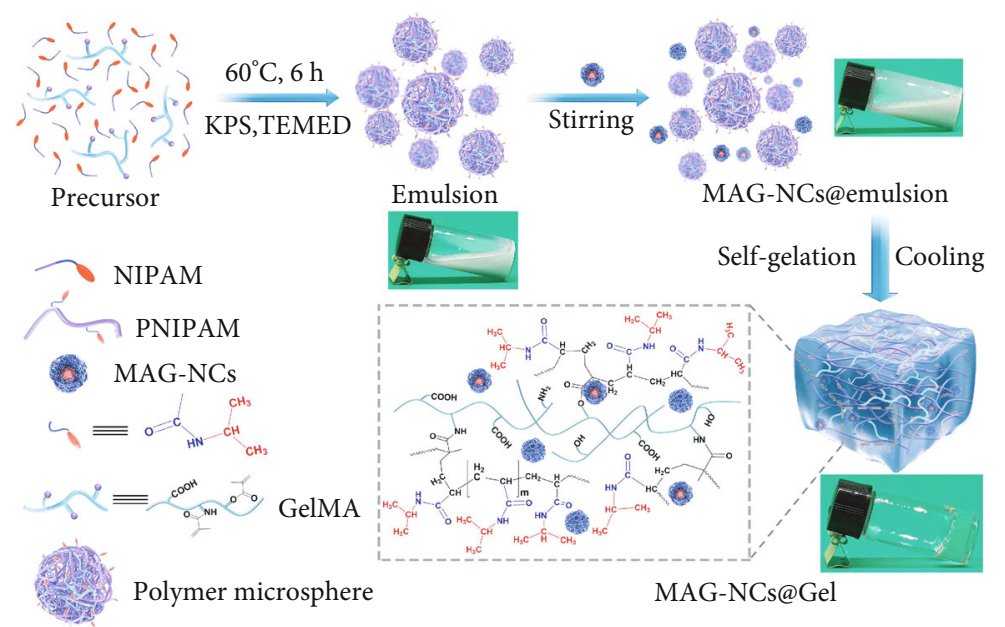

(a)

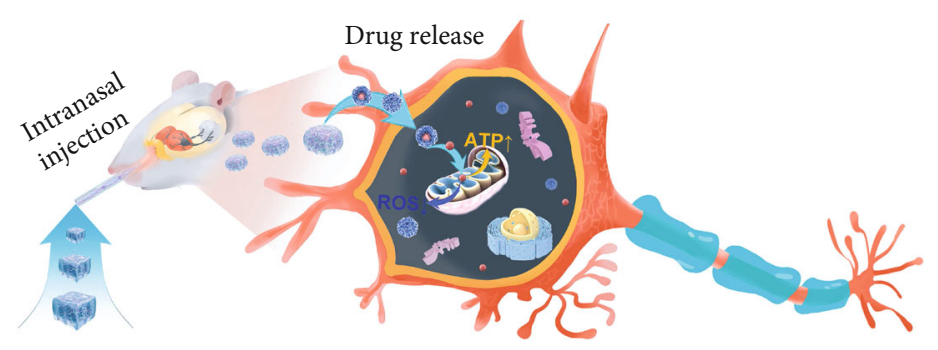

(b)

FIGURE 1: Synthesis and application of MAG-NCs@Gel. (a) Schematic diagram of MAG-NCs@Gel preparation and (b) the targeting treatment of PD via the intranasal injection of the hydrogel with release NCs.

without MAG-NCs (Figure S4). In addition, those MAGNCs were also clearly observed in SEM images of MAGNCs@Gel following a two-week storage at room temperature, suggesting that MAG-NCs@Gel preparations are highly stable (Figure S5). Besides, TEM images of MAG-NCs@Gel preparations show the size of MAG-NCs to range from 20 to $30 \mathrm{~nm}$, which was closed to that of the MAG-NC dispersion (Figure 1(b)). FT-IR spectra of MAGNCs@Gel displayed the presence of absorption peaks at $1635 \mathrm{~cm}^{-1}(\mathrm{C}=\mathrm{O}), 1530 \mathrm{~cm}^{-1}$ (N-H of amide), $1455 \mathrm{~cm}^{-1}$ $(\mathrm{C}-\mathrm{H})$, and $3291 \mathrm{~cm}^{-1}$ (N-H stretching vibration peak of GelMA) (Figure S6) [49]. Owing to the very low MAG-NC content in these samples $(<0.2 \mathrm{wt} \%)$, no characteristic peak of MAG-NCs was detectable. Rheological studies were then performed exploring the viscoelasticity and injectability of MAG-NCs@Gel [50, 51]. Based upon the dependence of the $G^{\prime}$ and $G^{\prime \prime}$ of MAG-NCs@Gel on the oscillation strain (Figure S7), the reversible gel state to flow state transition was determined to occur at $~ 80 \%$ strain (gel state: $G^{\prime}>G^{\prime \prime}$ and flow state: $G^{\prime}<G^{\prime \prime}$ ) [52]. Similarly, the gel-flow state transition of the blank hydrogel also occurred at $80 \%$ strain. Step-strain measurements with 5 times cycle revealed that $G^{\prime}$ was higher than $G^{\prime \prime}$ at low strain (1\%), whereas at higher strain levels (200\%), $G^{\prime}$ was lower than $G^{\prime \prime}$ (Figure S8). Those results suggested that the MAGNCs@Gel exhibited self-healing properties [53]. In frequency sweep analyses, the $G^{\prime}$ and $G^{\prime \prime}$ of MAG-NCs increased with increasing angular frequency, and $G^{\prime}$ was always greater than $G^{\prime \prime}$, so that gel characteristics were observed across the entire frequency range (Figure 2(c)). Relative to MAG-NCs@Gel, the $G^{\prime}$ and $G^{\prime \prime}$ of the blank hydrogel were smaller, although they were similarly dependent upon angular frequency (Figure S9). This suggests that interactions between MAG-NCs and polymer networks can enhance MAG-NCs@Gel viscoelasticity to some degree. MAG-NCs@Gel shear thinning was assessed based upon the association between the viscosity and shear rate (Figure 2(d)). The viscosity declines as the shear rate increases, suggesting that this hydrogel exhibits promising injectable properties (Figure 2(e), Movie S2) [54, 55]. In order to obtain the release performance of MAG-NCs@Gel

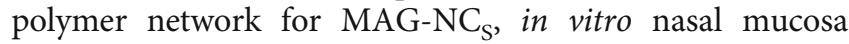
permeation assays were carried out, the results indicated that $\sim 100 \%$ of MAG-NCs were released from MAGNCs@Gel within $72 \mathrm{~h}$ (Figure 2(f)), in which the permeation efficiency was higher than the MAG group ( 30.7\%) and more durable release time as compared to the MAG-NCs group (Figure S10). These results suggested that MAG-NCs@Gel hydrogel loading significantly improved in vitro release and prolonged the overall drug release duration through the interaction between polymer and MAG-NCs to construct the suitable network structure. the neuroprotective properties of MAG and MAG-NC 


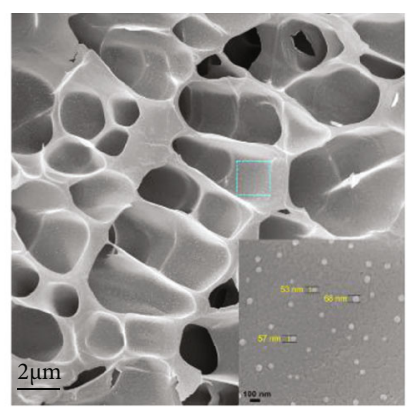

(a)

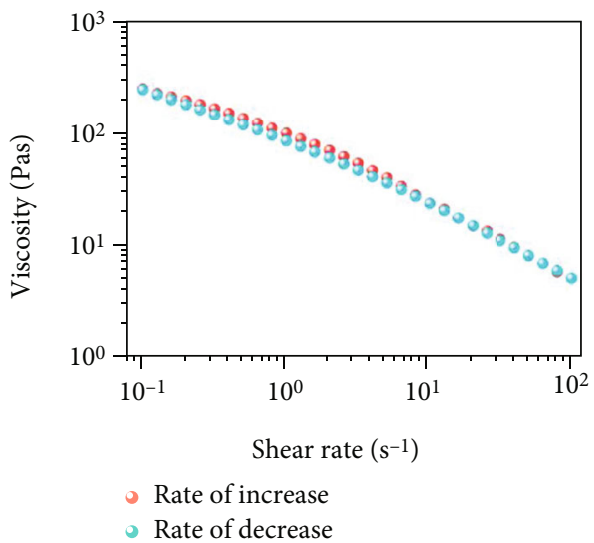

(d)

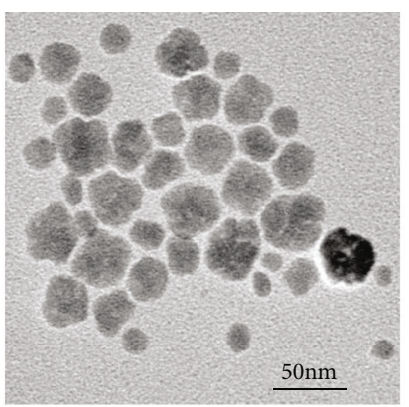

(b)

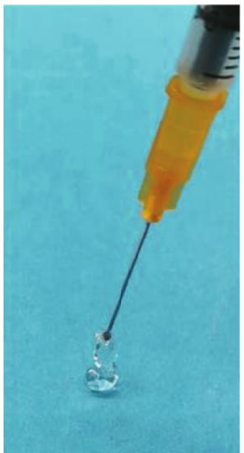

(e)

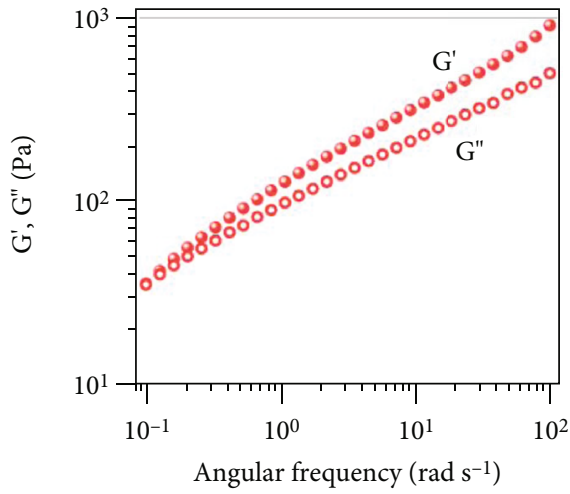

(c)

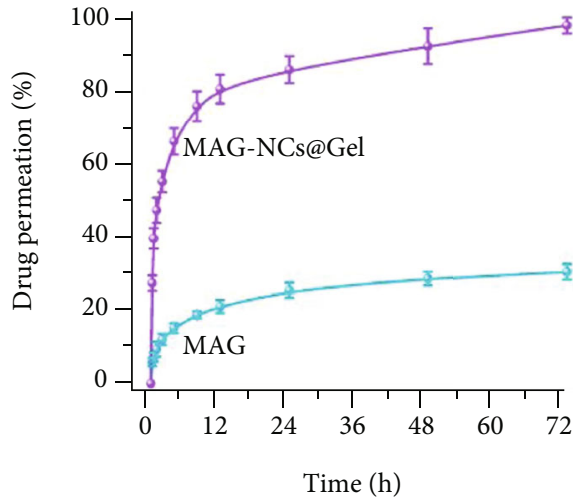

(f)

FIGURE 2: Structure characterization and performance measurement of MAG-NCs@Gel. SEM (a) and TEM (b) images of MAG-NCs@Gel preparations. (c) The dependence of $G^{\prime}$ and $G^{\prime \prime}$ of MAG-NCs@Gel on the angular frequency. (d) The association between MAG-NCs@Gel viscosity and shear rate. (e) Optical image of MAG-NCs@Gel injection. (f) Drug ex vivo nasal mucosa permeation curves as a function of time.

preparations within the context of $\mathrm{MPP}^{+}$-induced cytotoxicity, SH-SY5Y cell survival following treatment with these preparations was assessed via MTT assay [56]. At concentrations below $160 \mu \mathrm{M}$, no significant cytotoxicity was observed for MAG or MAG-NCs (Figure S11). Meanwhile, neuroprotection was evaluated by treating SH-SY5Y cells with MAG or MAG-NCs $(30 \mu \mathrm{M})$. Cells treated with $\mathrm{MPP}^{+}$ ( $2 \mathrm{mM}$ ) exhibited a survival rate of just $54.1 \%$ compared to the control group (100\% cell survival rate), whereas cells treated with MAG and MAG-NCs at the $30 \mu \mathrm{M}$ concentration exhibited respective viability values of $79.1 \%$ and $92.8 \%$ (Figure 3(a)). Even treatment with lower MAG and MAG-NC concentrations $(7.5 \mu \mathrm{M}$ or $15 \mu \mathrm{M})$ was associated with improved cell survival relative to the $\mathrm{MPP}^{+}$ group, although these effects were not as robust as those following treatment at the $30 \mu \mathrm{M}$ dose (Figure S12). Notably, the neuroprotective efficacy of MAG-NC preparations was superior to that of equivalent $\mathrm{MAG}$ concentrations. Consistently, CLSM images of SH-SY5Y cells stained implementing AM-PI (green-red) were prepared, with red and green fluorescence, respectively, corresponding to apoptotic and viable cells (Figure 3(b)). While control samples exhibited no evidence of cellular apoptosis, significant neurotoxic cell death was evident in the $\mathrm{MPP}^{+}$model group, whereas both the $30 \mu \mathrm{M}$ MAG-
NCs and $30 \mu \mathrm{M}$ MAG groups exhibited significant increases in the proportion of live cells, with this effect being more pronounced in the MAG-NCs group as compared to the MAG group. Flow cytometry analyses were further performed to quantify the neuroprotective effects of these preparations (Figure 3(c)), revealing respective frequencies of initial and tardy apoptotic cells in the MAG and MAG-NCs groups of $22.6 \%$ and $14.8 \%$, with these rates being higher than those in the control group $(\sim 5 \%)$, but much lower than those in the $\mathrm{MPP}^{+}$group ( $36.9 \%)$. Mitochondrial membrane potential measurements also yielded similar results (Figure 3(d)). Mitochondria are the main place for ATP production and an important organelle to promote cell energy conversion. The decrease of mitochondrial membrane potential is a landmark event in the early stage of apoptosis. The percentage of apoptotic cells in the control, $\mathrm{MPP}^{+}, \mathrm{MAG}$, and MAG-NCs groups was $5.86 \%, 36.43 \%, 23.1 \%$, and $15.1 \%$, respectively, confirming that MAG-NCs were associated with superior antiapoptotic efficacy.

2.3. Plasma and Brain Pharmacokinetics. MAG pharmacokinetic studies were performed in rats at a $1.0 \mathrm{mg} / \mathrm{kg}$ dose. In plasma pharmacokinetic analyses (Figure S13a), intranasally administered MAG-NCs@Gel was associated 


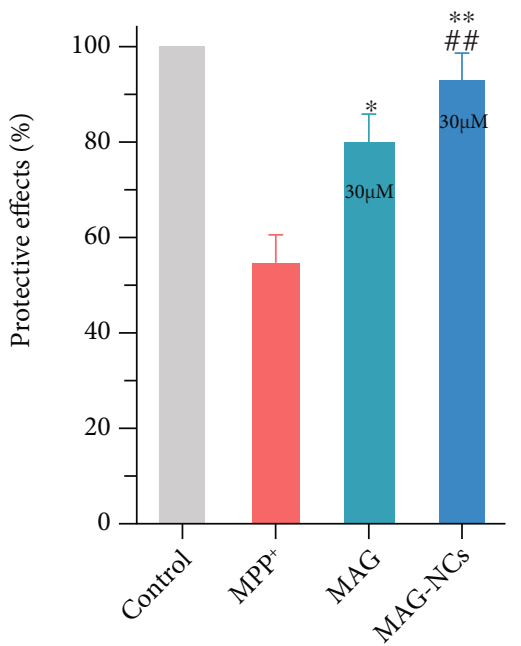

(a)

Control

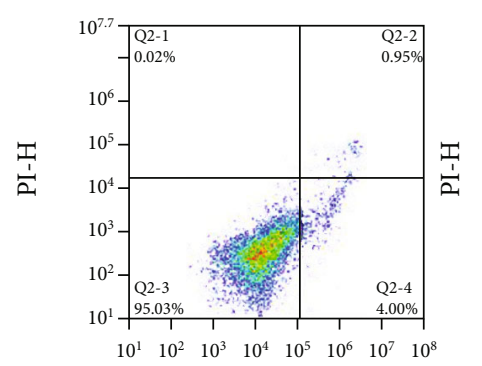

Annexin V FITC-H

Control

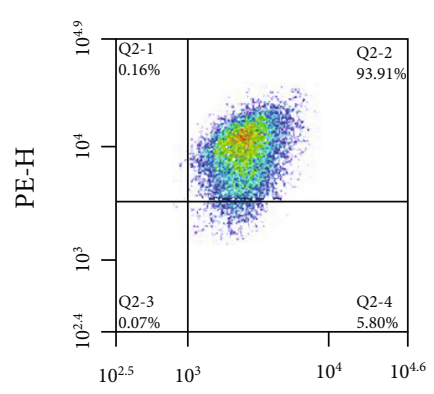

FITC-H

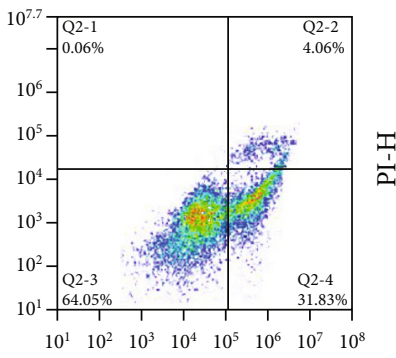

Annexin V FITC-H

$\mathrm{MPP}^{+}$

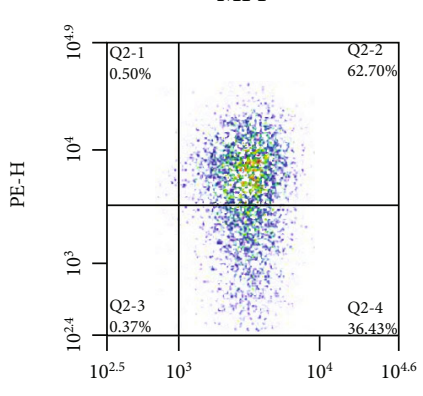

FITC-H
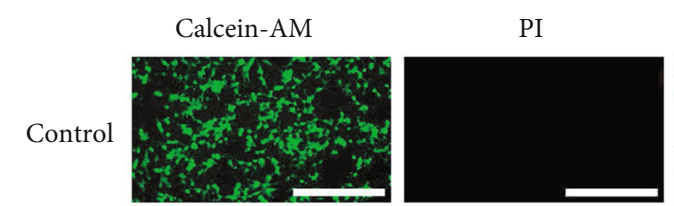

Merged

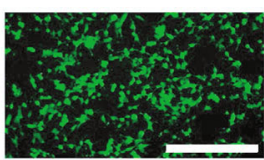

$\mathrm{MPP}^{+}$

MAG

$(30 \mu \mathrm{M})$
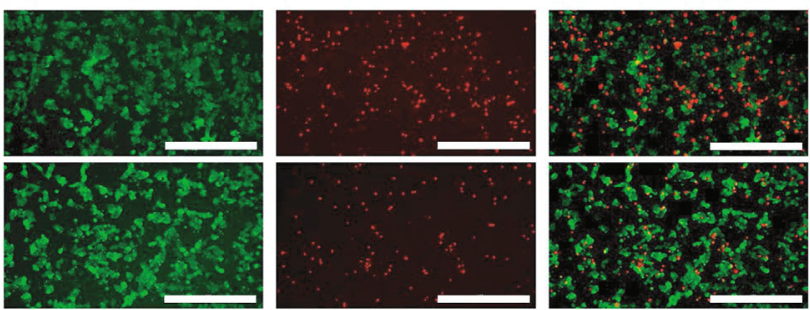

MAG-NCs

$(30 \mu \mathrm{M})$
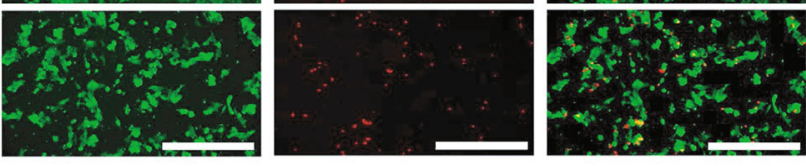

(b)

MAG $(30 \mu \mathrm{m})$

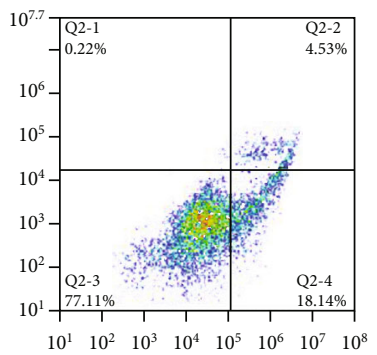

Annexin V FITC-H

(c)

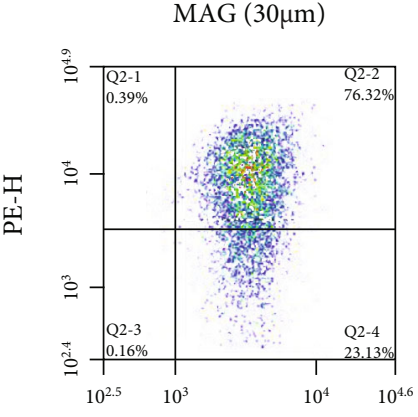

FITC-H
MAG-NCs $(30 \mu \mathrm{m})$

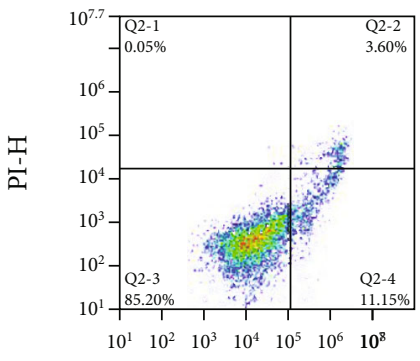

Annexin V FITC-H

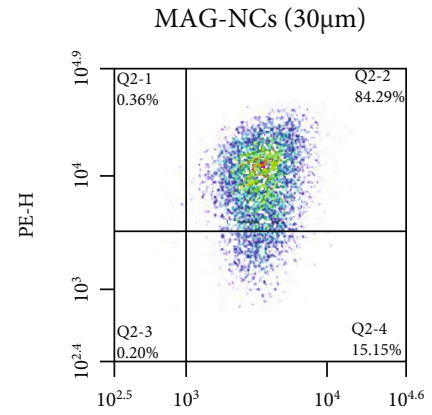

FITC-H

(d)

Figure 3: Protective effects of MAG-NCs. (a) Protective effects of MAG $(30 \mu \mathrm{M})$ and MAG-NCs (30 $\mu \mathrm{M})$ against MPP $^{+}$-induced cell death and cytotoxicity. ${ }^{*} p<0.05$ and ${ }^{* *} p<0.01$ vs. the $\mathrm{MPP}^{+}$group. ${ }^{*} p<0.05$ and ${ }^{\# \#} p<0.01$ vs. the MAG group at the same concentration. (b) Confocal laser scanning microscopy (CLSM) images of SH-SY5Y cells in the indicated treatment groups, with live and apoptotic cells being stained green (Calcein-AM) and red (propidiumiodide (PI)), respectively. Flow cytometry (c) and mitochondrial membrane potential (d) analysis of Annexin V FITC-H/PI-stained SH-SY5Y cells within the demonstrated treatment groups. Scale bars: $100 \mu \mathrm{m}$.

with sustained MAG detection in the plasma over $12 \mathrm{~h}$ postadministration, whereas plasma MAG levels in rats intravenously injected with MAG were only detectable in the first 3 hours (Table S1), and MAG-NC treatment prolonged the MAG circulation to the 6 hours. The MAGNCs@Gel group exhibited a MAG $T_{\max }$ of $3.50 \pm 0.55 \mathrm{~h}$ and $C_{\max }$ of $25.13 \pm 3.87 \mathrm{ng} / \mathrm{mL}$, whereas for MAG-NCs these values were $0.083 \mathrm{~h}$ and $128.87 \pm 18.06 \mathrm{ng} / \mathrm{mL}$, respectively (Table S2), indicating that intranasal MAGNCs@Gel administration is associated with sustained and controlled drug release. Furthermore, the $T_{1 / 2}$ of the MAG-NCs@Gel group $(3.05 \pm 0.41 \mathrm{~h})$ was over 2 -fold 
higher than that in both the MAG and MAG-NCs groups, consistent with the prolongation of plasma release time for MAG-NCs loaded into PNIAM hydrogel as compared to free MAG-NCs. Consistently, 10.14-fold and 3.34-fold increase in the $\mathrm{MRT}_{0-t}$ value for MAG were detected in rats in the MAG-NCs@Gel group $(5.68 \pm 0.47 \mathrm{~h})$ relative to the $\mathrm{MAG}_{i . v \text {. group }}(0.56 \pm 0.15 \mathrm{~h})$ and MAG-NCs group $(1.70 \pm 0.22 \mathrm{~h})$, respectively. Furthermore, the $\mathrm{AUC}_{0-t}$ in MAG-NCs@Gel-treated animals was $302.07 \pm 29.86$ $\mathrm{ngh} \mathrm{mL} \mathrm{m}^{-1}$, which was 7.47 times, 5.92 times, and 3.38 times higher than that observed for rats in the $\mathrm{MAG}_{i . v}$. group $\quad\left(40.43 \pm 4.17 \mathrm{ngh} \mathrm{mL}^{-1}\right), \quad \mathrm{MAG}_{i . n .} \quad(50.97 \pm 6.09$ $\left.\mathrm{ngh} \mathrm{mL}{ }^{-1}\right)$, and MAG-NCs group $(89.27 \pm 10.23 \mathrm{ngh} \mathrm{mL}$ $\left.{ }^{1}\right)$. These results thus indicated that MAG-NCs improved the bioavailability of MAG, and MAG-NCs loaded with a thermosensitive hydrogel may be associated with better gradual MAG release, protecting against rapid loss of drug and facilitating effective drug release.

Direct delivery of medications to the brain is one of the most promising approaches to effectively treating PD. Therefore, drug pharmacokinetics within the brain is a key consideration when evaluating potential PD therapeutics. The association between MAG concentrations and time in the brain was thus assessed following its intranasal or intravenous administration (Figure S13b), and related pharmacokinetic parameters were calculated. The $T_{\max }$ value in animals in the MAG-NCs@Gel group (4.67 \pm 1.03 h) was 9.34 times and 7.4 times that of animals in the $\mathrm{MAG}_{i . v .}$ group $(0.5 \pm 0 \mathrm{~h})$ and MAG-NCs group $(0.63 \pm 0.31 \mathrm{~h})$, respectively. The $C_{\max }$ for the MAGNCs@Gel group was also markedly increased relative to the $\mathrm{MAG}_{i . v \text {. }}$ MAG $\mathrm{MA}_{i . n \text {. }}$ and MAG-NCs groups $(131.75 \pm 10.21 \mathrm{ng} / \mathrm{g}$ vs. $22.85 \pm 3.94,33.01 \pm 4.03$, and $34.76 \pm 3.19 \mathrm{ng} / \mathrm{g})$. The $T_{1 / 2}(11.51 \pm 1.42 \mathrm{~h})$ and $T_{\max }$ of the MAG-NCs@Gel group were also longer in the brain relative to the plasma, suggesting that the majority of administered MAG was effectively absorbed within the brain. The $\mathrm{AUC}_{0-t}$ in the MAG-NCs@Gel group was $2555.69 \pm 336.17 \mathrm{nghg}-1$, with this value being 27.4 -fold higher than that in the MAG-NCs group (93.24 \pm 10.96 ngh $\mathrm{g}^{-1}$ ). In summary, these results suggested that continuous drug delivery and effective $\mathrm{BBB}$ penetration were achieved in the MAG-NCs@Gel group by utilizing controlled-release MAG-NCs encapsulated within a PNIPAM hydrogel. These data indicated that MAGNCs@Gel with DTE\% and DTP\% values of 809.98\% and $87.65 \%$ were able to efficiently deliver MAG to the brain.

\subsection{Treatment of MPTP-Induced Behavioral Deficits. To} confirm the satisfactory therapeutic efficacy of MAGNCs@Gel as a treatment for the behavioral deficits of MPTP-induced mice, a series of comprehensive experiments was performed (Figure 4(a)). Initially, MPTP was administered for mice $\left(18 \mathrm{mg} \mathrm{kg}^{-1}\right)$, resulting in significant motor behavioral deficits [57]. The effects of intranasal MAGNCs@Gel administration on these deficits were then assessed, with mice being examined using the rotarod, pole, and open field tests seven days later. On day eight of this study, mice were then euthanized, and immunohistochemi- cal staining for DA and its metabolites, blood analyses, and tests of hepatic and renal functionality were conducted. MPTP induces motor symptoms including impaired coordination and balance, bradykinesia, and diminished spontaneous motor performance. In rotarod tests, mice treated with MAG or L-DOPA exhibited significant improvements relative to MPTP-treated model mice (Figure 4(b)), with the values of time to reach the bottom ( $T$-total) and time to turn ( $T$-turn $)(8.35 \pm 0.71 \mathrm{~s}$ and $1.73 \pm 0.15 \mathrm{~s})$ in the MAGNCs@Gel group being lower than those in the MAG group $(3.37 \pm 0.27 \mathrm{~s}$ and $13.31 \pm 1.01 \mathrm{~s})$ and the MAG-NCs group $(2.81 \pm 0.20 \mathrm{~s}$ and $11.08 \pm 0.94 \mathrm{~s})$, consistent with the optimal therapeutic efficacy of MAG-NCs@Gel preparations. Relative to other MAG treatments, the MAG-NCs@Gel group exhibited similar improvements in the pole test (Figure 4(c)), wherein MAG-NCs@Gel-treated mice exhibited prolonged fall latency and a reduced number of drops. There was also a significant increase in average travel distance and speed in the MAG-NCs@Gel group relative to other experimental groups in the open field test (Figures 4(d) and 4(e)). In summary, these behavioral tests indicated that MAG-NCS@Gel treatment was associated with excellent therapeutic efficacy as a treatment for MPTP-induced motor dysfunction, which was comparable to that detected in mice treated with L-DOPA.

2.5. The Prevention of $\mathrm{TH}^{+}$Neuron Loss and Reversal of Mitochondrial Dysfunction. MPTP-induced neurotoxicity results in the loss or death of $\mathrm{TH}^{+}$neurons in the midbrain substantia nigra together with reductions in levels of homovanillic acid (HVA) and striatal DA, 3,4-dihydroxyphenylacetic acid (DOPAC). Fluorescent images of $\mathrm{TH}^{+}$neurons clearly emphasized the protective influences of MAGNCs@Gel treatment against MPTP-induced neurotoxicity, with these results being better than those in the MAG or MAG-NCs groups (Figure 5(a)). Notably, the protective efficacy in the MAG-NCs@Gel group and the L-DOPA group were similar, with respective protection levels that were $92.94 \pm 7.66 \%$ and $91.59 \pm 7.84 \%$ of those in the control group, respectively (Figure $4(\mathrm{~b})$ ). In addition, MPTP treatment rapidly reduced striatal DA, DOPAC, and HVA levels, whereas all experimental treatments significantly increased these levels. The protective effect on neurons of MAG-NCs had been confirmed in the in vitro experiment. Importantly, these improvements were most robust in the MAGNCs@Gel group relative to other MAG treatment groups (Figure S14), indicating that this drug release platform was able to effectively protect against MPTP-induced $\mathrm{TH}^{+}$ neuron death and loss while improving the DA and DA metabolites levels within the striatum.

One of the most valuable noninvasive imaging tools for monitoring changes in the cerebral blood flow in vivo is PET, and it is thus widely used to diagnose PD and other neurodegenerative diseases, including those where in neuronal dysfunction precedes structural change. In such analyses, ${ }^{18} \mathrm{~F}-\mathrm{FDG}$ is primarily employed to trace the utilization of glucose within the brain and associated PD-related metabolic changes. In this study, ${ }^{18}$ F-FDG PET/CT imaging was conducted to assess cerebral glucose metabolism in mice 


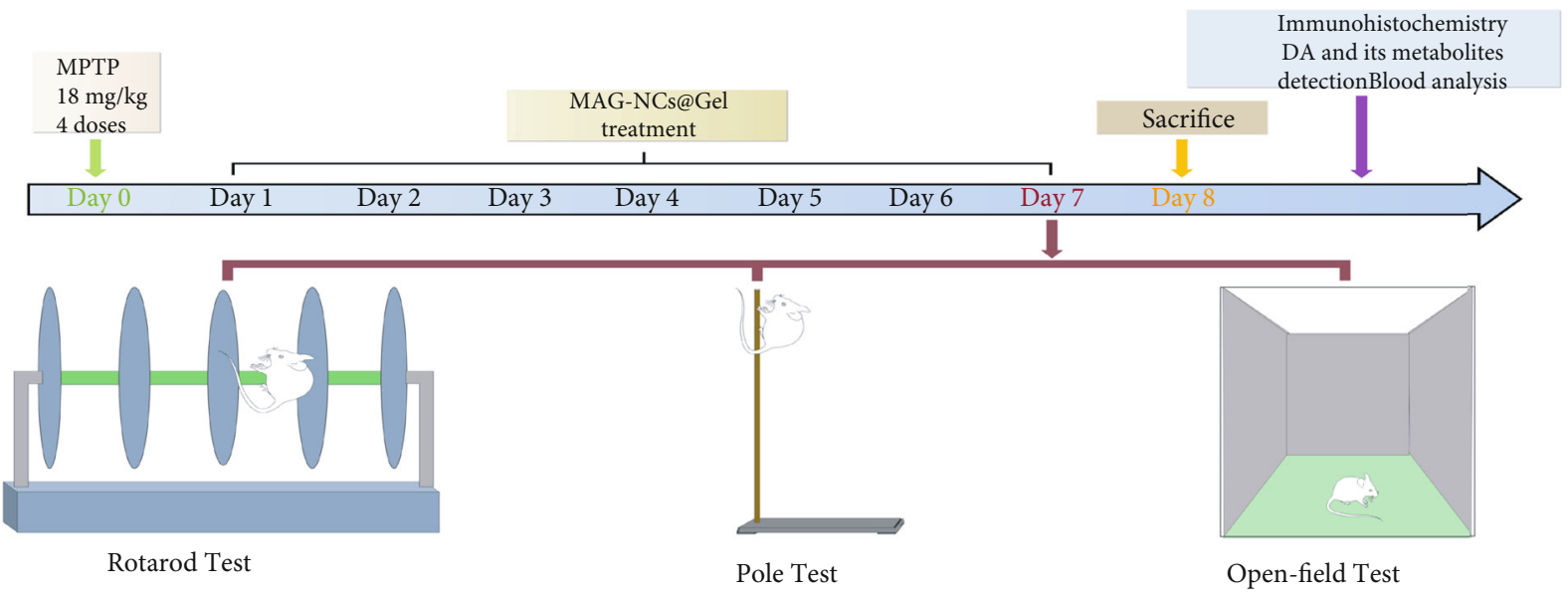

(a)
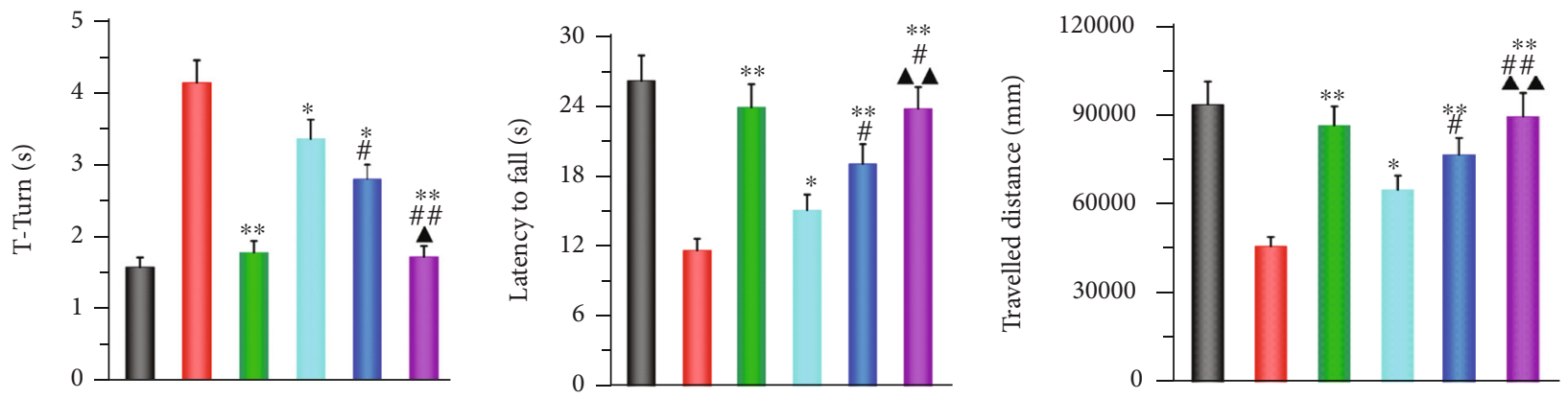

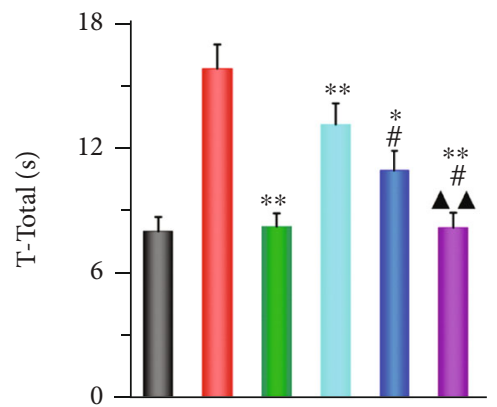

(b)
Control

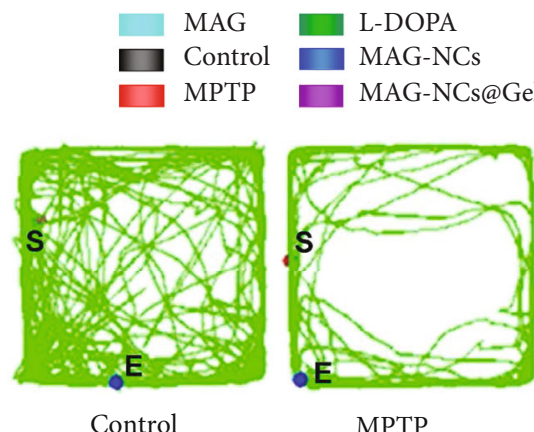

MPTP

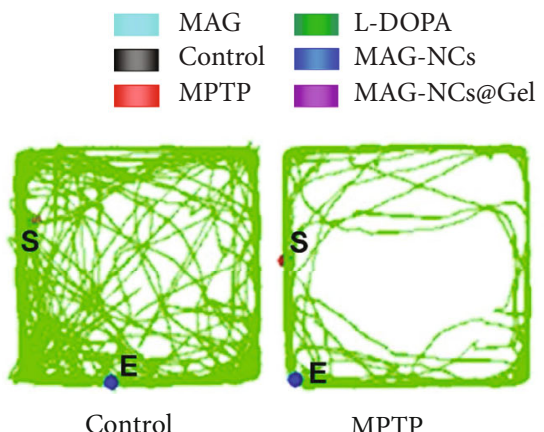

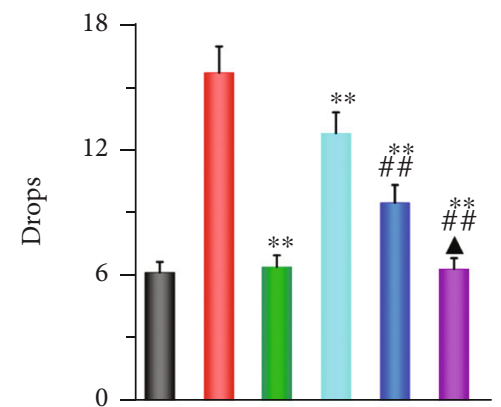

(c)

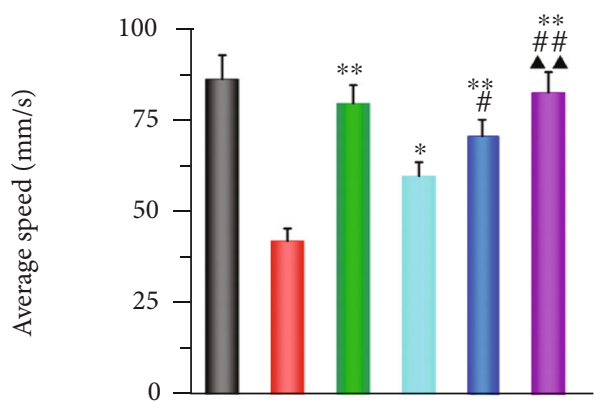

(d)

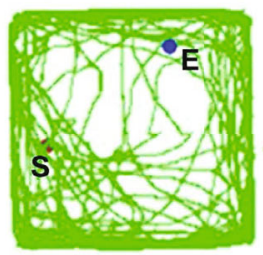

L-DOPA

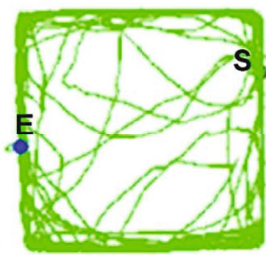

MAG

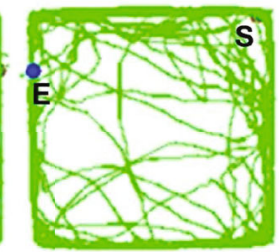

MAG-NCs

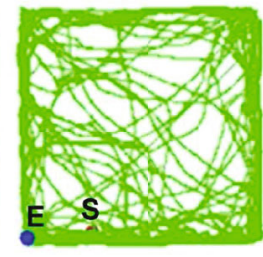

MAG-NCs@Gel

(e)

FIGURE 4: Behavioral analysis. (a) Schematic overview of the comprehensive experimental procedure for the MAG-NCs@Gel treatment of MPTP-induced PD. Murine behavioral analyses were conducted: (b) the rotarod test: T-turn (A) and T-total (B), (c) the pole test: latency to fall (A) and drops (B), and (d) the open field test: average travel distance (A) and speed (B). (e) Representative activity paths for mice in the indicated groups as measured via digital tracking (red: starting position; blue: ending position). ${ }^{*} p<0.05$ and ${ }^{* *} p<0.01$ vs. the MPTP group. ${ }^{\#} P<0.05$ and ${ }^{\# \#} P<0.01$ vs. the MAG group. ${ }^{\boldsymbol{\Delta}} p<0.05$ and ${ }^{\mathbf{\Delta}} p<0.01$ vs. the MAG-NCs group. 

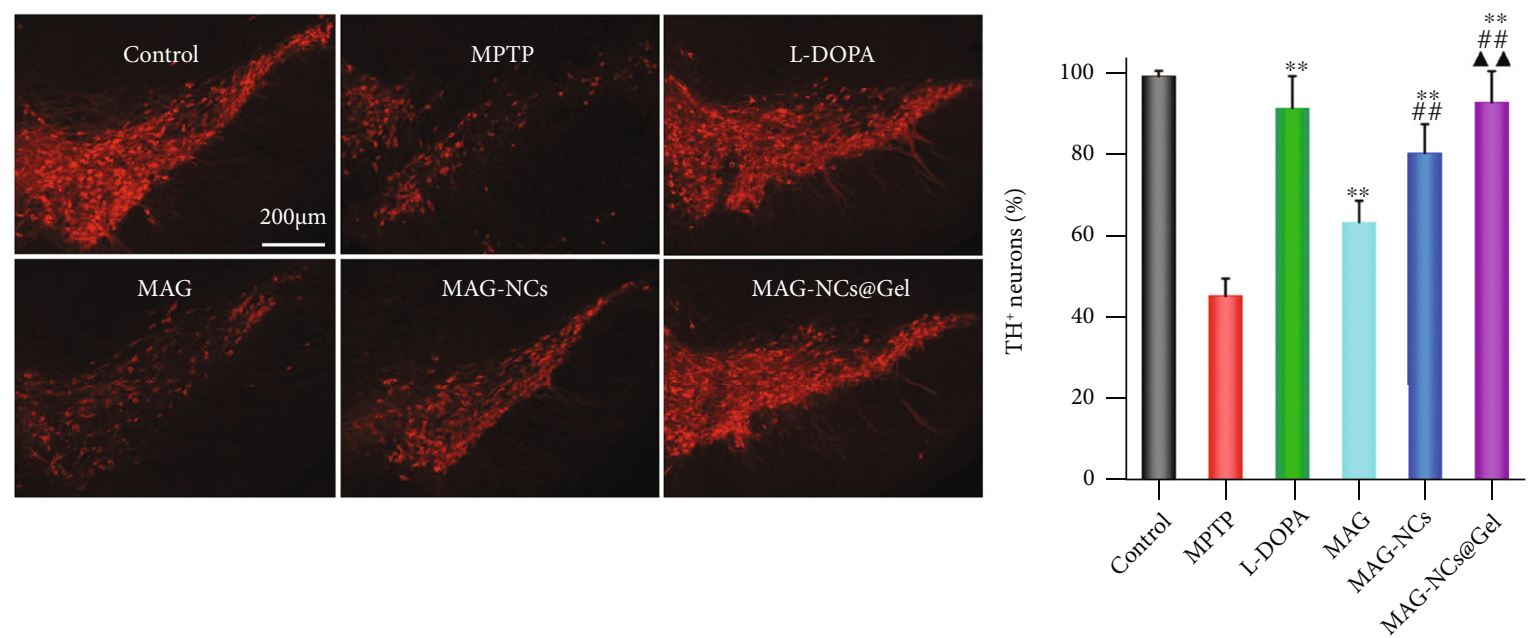

(a)

(b)
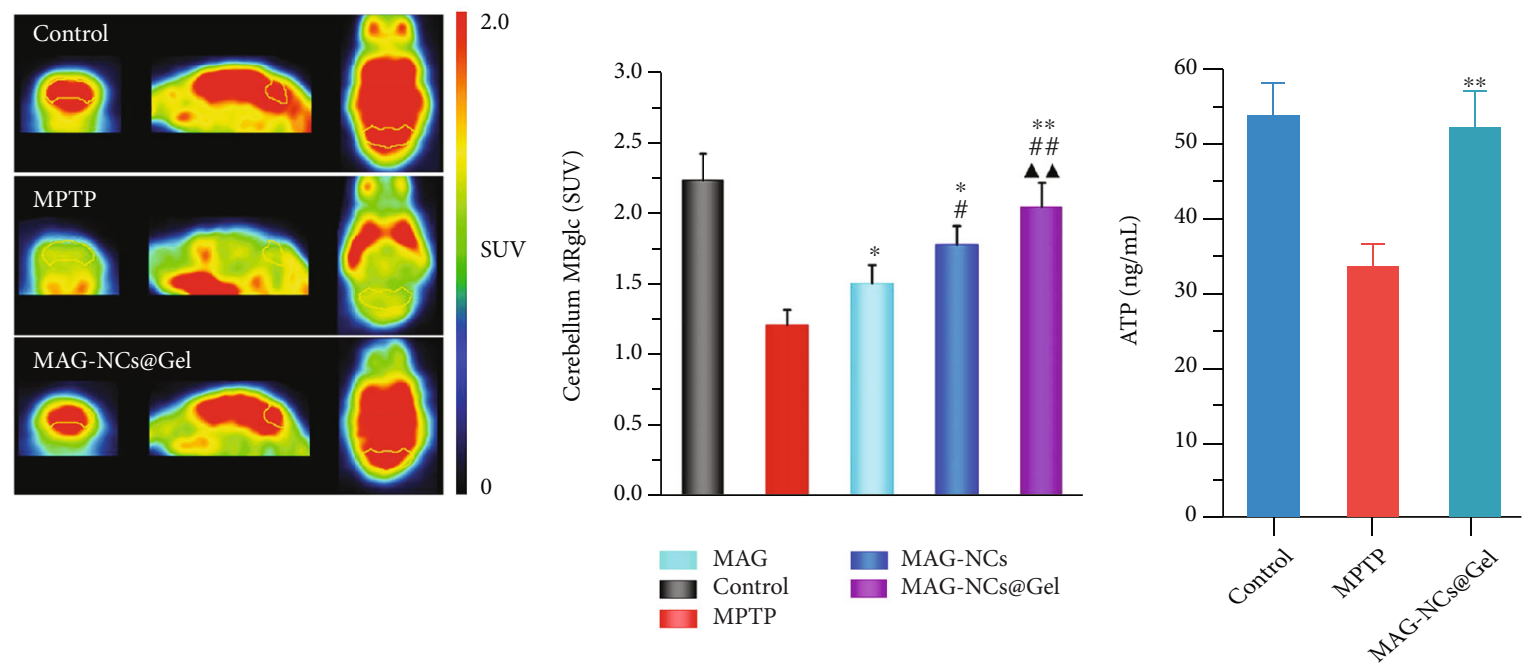

(c)

(d)

(e)

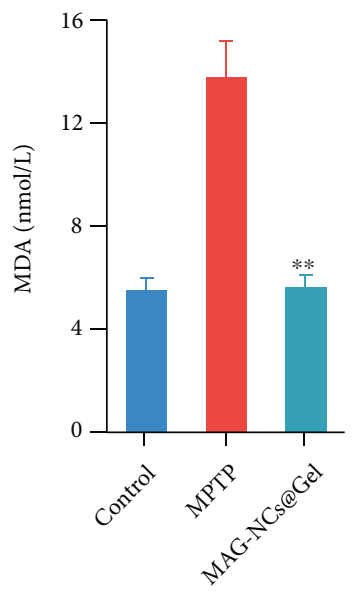

(f)

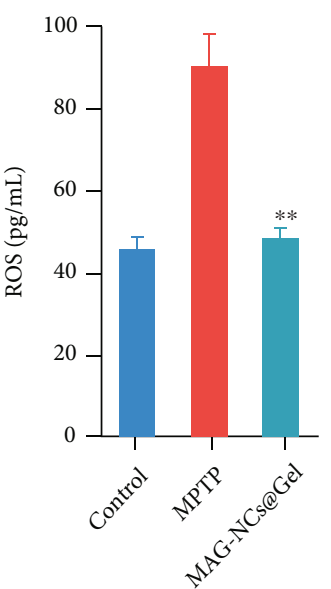

(g)

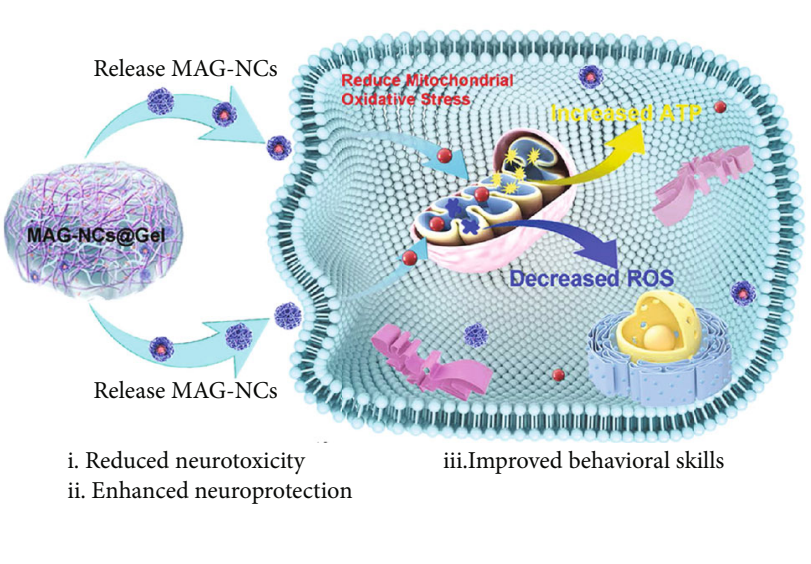

(h)

FIgure 5: Targeted treatment of PD with MAG-NCs@Gel. (a) Images of $\mathrm{TH}^{+}$immunofluorescent-stained sections. (b) Assessment of TH ${ }^{+}$ neurons in the substantia nigra. (c) ${ }^{18}$ F-FDG PET images of brain anatomy in the coronal, sagittal, and transverse directions. (d) ${ }^{18} \mathrm{~F}-\mathrm{FDG}$ uptake values in the left striatum for the indicated groups. Mitochondrial functional parameters detecting in the midbrain, including (e) ATP levels, (f) MDA levels, and (g) ROS levels. (h) A schematic overview of the mechanistic treatment of mitochondrial dysfunction using the MAG-NCs@Gel controlled release platform. ${ }^{*} p<0.05$ and ${ }^{* *} p<0.01$ vs. the MPTP group. ${ }^{\#} p<0.05$ and ${ }^{\# \#} p<0.01$ vs. the MAG group. ${ }^{\mathbf{\Delta}} p<0.05$ and ${ }^{\mathbf{\Lambda}} p<0.01$ vs. the MAG-NCs group. 


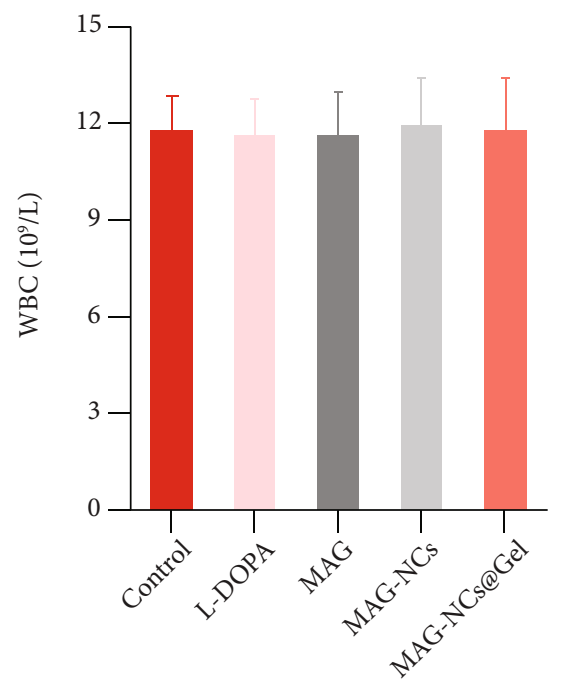

(a)

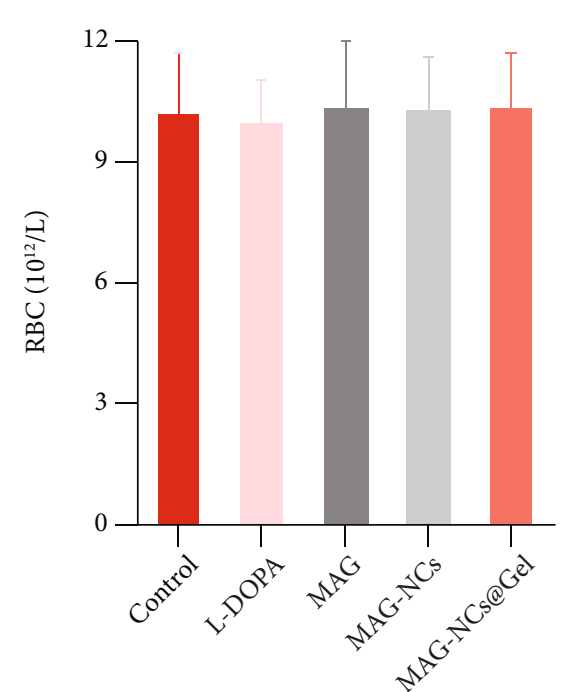

(b)

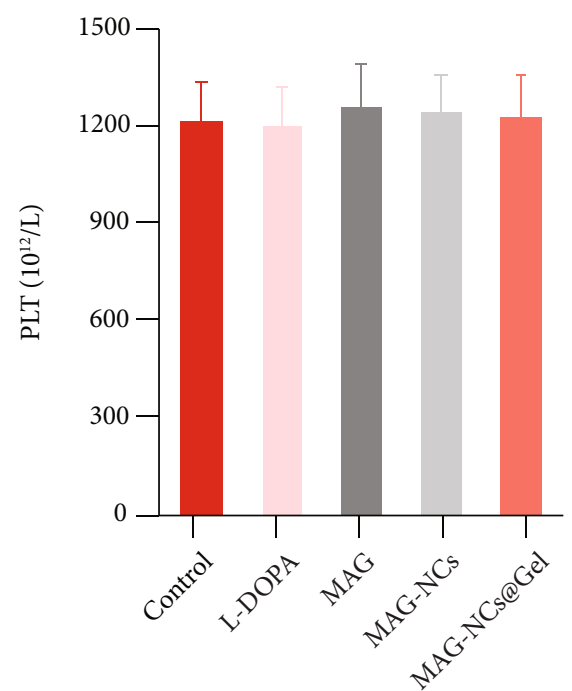

(c)

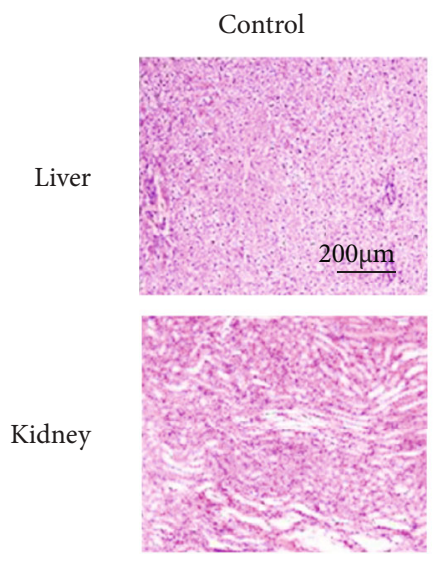

L-DOPA

MAG
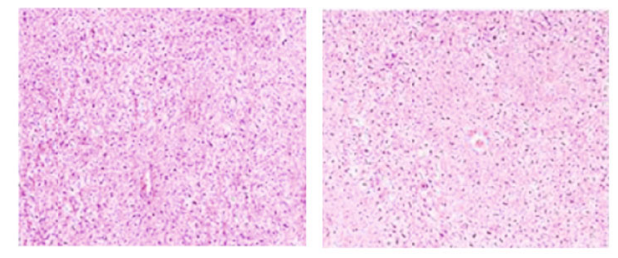

MAG-NCs

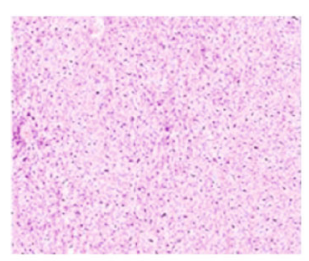

MAG-NCs@Gel
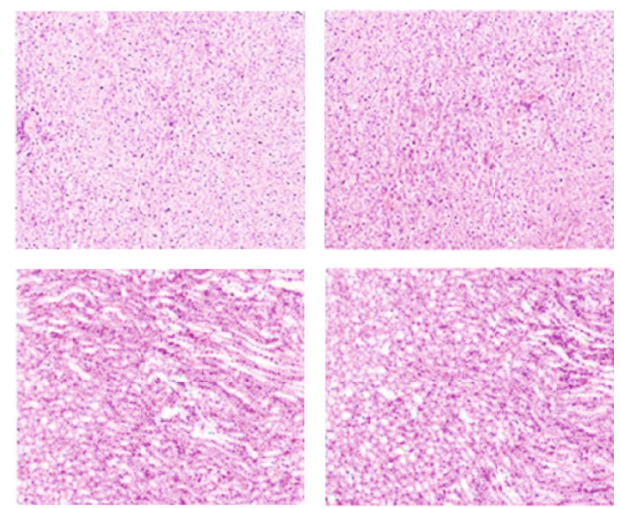

(d)

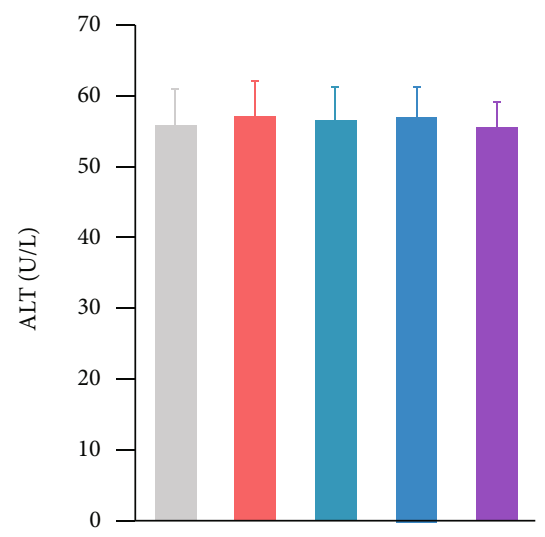

(e)

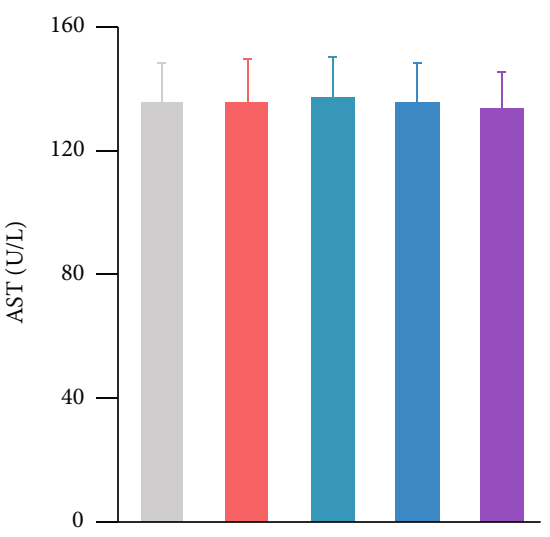

(f)

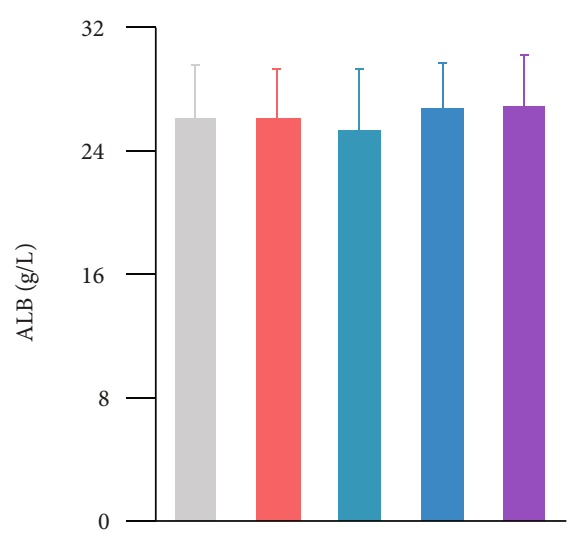

(g)

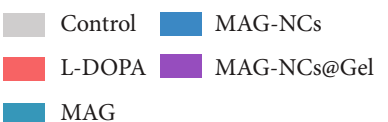

Figure 6: Biocompatibility analysis. Assessment of in vivo biocompatibility in different treatment groups. Routine blood analyses of (a) WBC, (b) RBC, and (c) (PLT). (d) Representative H\&E-stained liver and kidney tissue sections. Liver and kidney functional parameters were measured, including (e) ALT, (f) AST, and (g) ALB. 
with MPTP-induced PD on day 7. Relative to control animals, those in the treated MPTP group exhibited significantly decreased ${ }^{18}$ F-FDG uptake, consistent with metabolic abnormalities (Figure 5(c)). However, such energy metabolism was clearly restored to levels close to those in control animals following MAG-NCs@Gel treatment. The energy metabolism levels in these mice were improved to some degree in the MAG and MAG-NCs groups as well, although the effects were not as robust as those in the MAG-NCs@Gel group (Figure S15). When further assessing changes in cerebellar glucose metabolism (MRglc), the cerebellar MRglc levels of mice in the MAGNCs@Gel group remained at $2.058 \pm 0.171$ and were close to the levels in control animals and higher than levels in animals in any other experimental treatment groups (Figure 5(d)). Changes in body weight over time following drug treatment can additionally reflect the restoration of normal MRglc. Relative to the steady weight gain observed in the control group, mice in the L-DOPA or MAG group initially exhibited some degree of weight loss followed by gradual weight increases, consistent with MRglc recovery (Figure S16). To establish the protective effects of this MAG-NCs@Gel sustained-release platform on mitochondrial function within the midbrain MAG-NCs platform on midbrain mitochondria, MPTP-induced PD model animals were again utilized. In this model system, MPTP is converted into toxic $\mathrm{MPP}^{+}$within dopaminergic neurons, wherein it agglomerates within the mitochondrial and blocks complex I of the electron transport chain. Mitochondrial function in treated animals was assessed by measuring ATP, ROS, and malondialdehyde (MDA) levels. As demonstrated in Figure 5(e), the level of ATP in the MPTP group $\left(33.54 \pm 2.98 \mathrm{ng} \mathrm{mL}^{-1}\right)$ decreased significantly relative to the control group $\left(53.64 \pm 4.33 \mathrm{ng} \mathrm{mL}^{-1}\right)$. Relative to the MPTP group, the ATP levels in the MAGNCs@Gel group $\left(52.13 \pm 4.82 \mathrm{ng} \mathrm{m}^{-1}\right)$ significantly increased, approaching levels in the control and L-DOPA groups (Figure S17a). ROS levels in MPTP model animals were roughly twofold higher than those in control animals (Figure 5(f)), while levels of the oxidative stress indicator MDA were similarly increased in the MPTP group (Figure 5(g)). Following MAG-NCs@Gel treatment, ROS and MDA levels returned to normal, consistent with the excellent therapeutic efficacy of this therapeutic platform within this murine model system owing to its ability to efficiently reverse MPTP-induced mitochondrial dysfunction. MDA and ROS levels were also decreased in other treatment groups, but these effects were not as pronounced as in the MAG-NCs@Gel group (Figure S17b and c). The mechanism whereby this MAG-NCs@Gel release platform can treat mitochondrial dysfunction is summarized in Figure 5(h). Initially, MAG-NCs are continuously released from the PNIPAM hydrogel and can readily pass through the $\mathrm{BBB}$ whereupon they enter dopaminergic neurons via endocytosis to regulate mitochondrial ATP, ROS, and MDA levels so that they return to normal, thereby abrogating MPTP-induced neurotoxicity and facilitating neuroprotection. The behavioral skills of animals in these studies improved significantly to near baseline levels, thus confirming the effective treatment of PD in these animals.

2.6. In Vivo Biocompatibility Analysis. Following a 7-day treatment period, MAG-NCs@Gel biocompatibility following intranasal administration was assessed by examining nasal mucosal sections, performing routine blood analyses, and assessing organ inflammation or lesions. Relative to samples from the control group, no evidence of inflammation or structural damage was observed in samples from animals treated with blank hydrogels or different MAG treatments (Figure S18). Additionally, no differences in routine blood analysis parameters (WBC, RBC, and PLT) were observed in the MAG treatment groups relative to the control or L-DOPA treatment groups (Figures 6(a)-6(c)). Consistently, routine blood parameters (HGB, MCV, $\mathrm{MCH}, \mathrm{MCHC}$, and HCT) were also normal in these animals (Figure S19). H\&E-stained sections of major tissues including the liver and kidneys exhibited no evidence of inflammation or injury in any treatment group (Figure 6(d)), with no evidence of inflammation in the heart, lung, brain, or spleen sections from animals in the MAG-NCs@Gel group or other treatment groups (Figure S20). Given the synthetic nature of the PNIAM hydrogel, there is an associated risk of organ rejection or dysfunction, but no such abnormalities were observed when assessing liver and kidney function in treated mice (Figures 6(e)-6(g) and Figure S21). Therefore, these data suggested that our MAG-NCs@Gel sustained-release system exhibited satisfactory biocompatibility following intranasal injection, making it of potential value for the treatment of PD.

\section{Discussion}

In summary, this study developed a thermosensitive PNIPAM hydrogel for encapsulating MAG-NCs to facilitate sustained drug delivery following intranasal administration, thereby effectively treating a murine model of PD owing to associated self-gelation processes. It is needed to point out that the presence of MAG-NCs enhances the stability and strength of PNIPAM hydrogel, indicating the synergic action of weak interactions among MAG-NCs and PNIPAM chains. The LCST behavior of the drug delivery platform prolonged the residence time of MAG-NCs in the nasal cavity so that these MAG-NCs were able to readily cross the BBB to achieve the targeted regulation of ROS and ATP levels in dopaminergic neurons in the context of MPTP-induced mitochondrial dysfunction.MAG-NCs@Gel treatment was associated with significant reductions in dopaminergic neuron neurotoxicity in PD model mice, with concomitant improvements in neuroprotection and motor behavior. Importantly, this drug delivery platform also exhibited satisfactory biocompatibility without any evident side effects.

Together, these results underscore the potential value of intranasal MAG-NCs@Gel injection for the treatment of $\mathrm{PD}$, overcoming key limitations to traditional drug delivery such as the rapid loss of drugs from the nasal cavity and the restricted capability of drugs to cross the BBB. In 
addition, the work may realize controlled transmembrane of drug NCs and targeted treatment for Parkinson's disease, Alzheimer's disease, and other neurodegenerative diseases.

\section{Data Availability}

All data needed of this study are available in the article and its Supplementary Information files.

\section{Conflicts of Interest}

The authors declare no competing financial interest.

\section{Authors' Contributions}

Y. Tan and Y. Liu conceived the idea, designed the experiments, performed the experiments and the data analysis, and wrote original draft. Y. J. Liu, R. Ma, J. S Luo, H. J. Hong, X. J. Chen, S. P. Wang, and C. T. Liu performed the data analysis and investigation. Y. Zhang and T. K. Chen contributed to writing and editing the manuscript, provided conceptualization and methodology, and found funding support. All authors have given approval to the final version of the manuscript. Yun Tan and Yao Liu contributed equally to this work.

\section{Acknowledgments}

This work was supported by the National Natural Science Foundation of China (21773311 and 21972169), the Hunan Provincial Science and Technology Department Project (No. 2019TP1001), the Guangdong Basic and Applied Basic Research Foundation (2019B1515120043), the Research Fund of University of Macau (File no. MYRG2019-00121ICMS and MYRG2018-00207-ICMS), the Science and Technology Development Fund, Macau SAR (File no. 0098/2020/A), and the Key Project of Basic Research of Shenzhen (JCYJ20200109113603854).

\section{Supplementary Materials}

Supplementary 1. Materials and Methods: reagents, preparation of MAG-NCs, preparation of MAG-NCs@Gel, characterizations, and analysis of hydrogel viscoelasticity. Cell and animal experiments: ex vivo nasal mucosa permeation, cytotoxicity assessment, analysis of in vitro neuroprotective effect, flow cytometry, pharmacokinetics, therapeutic effects on MPTP-induced PD mouse model, behavioral evaluations, PET/CT imaging, immunofluorescence staining, determination of DA level in striatum, evaluation of mitochondrial function in midbrain, and in vivo biocompatibility analysis. Figure S1: (a) dependence transparency of hydrogels on temperature. (b) Estimating the LCST of hydrogels through differentiation of transparency to temperature. Figure S2: the temperature dependence of MAG-NCs@Gel viscoelasticity. Figure S3: particle size distribution (a) and TEM image (b) of MAG-NCs, (c) particle size of MAG-NCs for different storage times, and (d) TEM image of MAG-NCs following storage for 14 days at 40 C. Figure S4: SEM images of freeze-dried blank hydrogel. Figure S5: SEM images of freeze-dried MAG-NCs@Gel following storage for 14 days at room temperature. Figure S6: FT-IR of MAG-NCs@Gel. Figure S7: rheological properties of hydrogels as a function of oscillation strain. Figure S8: evaluation of injectability based on changes of $G^{\prime}$ and $G^{\prime \prime}$ of MAG-NCs@Gel under low (1\%) and high (200\%) oscillatory shear strain. Figure S9: the dependence of $G^{\prime}$ and $G^{\prime \prime}$ of blank hydrogel on angular frequency. Figure S10: ex vivo nasal mucosa permeation curve for MAG-NCs as a function of time. Figure S11: cell viability following incubation with different concentrations of treatment groups. Figure S12: drug-mediated protection against $\mathrm{MPP}^{+}$-induced cell death and cytotoxicity. ${ }^{*} p<0.05$ and ${ }^{\# \#} p<0.01$ vs. the MAG group. Figure S13: MAG-NCs and MAG-NCs@Gel pharmacokinetics in mice. (a) Plasma and (b) brain concentration-time profiles ( $n=6$ and 4, respectively). Figure S14: striatum parameters (c) DA, (d) DOPAC, and (e) HVA. ${ }^{*} p<0.05$ and ${ }^{* *} p<0.01$ vs. the MPTP group, ${ }^{\#} p<0.05$ and ${ }^{\# \#} p<0.01$ vs. the MAG group, $\boldsymbol{\Delta}_{p}<0.05$ and ${ }^{\boldsymbol{\Lambda}} p<0.01$ vs. the MAG-NCs group. Figure S15: ${ }^{18}$ F-FDG PET images of brain anatomy in the coronal (left), sagittal (middle), and transverse directions (right). (a) MAG group. (b) MAG-NCs group. Figure S16: body weight tracking for MPTP-inducted PD mice in the indicated treatment groups over a 7-day period. Figure S17: mitochondrial function parameters for different treatment groups were detected in the midbrain, (a) ATP level, (b) MDA level, and (c) ROS level. ${ }^{\#} p<0.05$ and ${ }^{\# \#} p<0.01$ vs. the MAG group. Figure S18: nasal mucosal sections from different groups. Figure S19: routine blood analysis parameters. Figure S20: representative images of $H \& E$ stained organs. Figure S21: quantitative assessment of liver and kidney function parameters. Table S1: plasma and brain pharmacokinetic criteria associated with MAG-NCs and MAG-NCs@Gel. Table S2: plasma and brain pharmacokinetic criteria associated with intravenous administration (MAG-NCs) and intranasal administration (MAG-NCs@Gel) ( $n=6$ or 4).

Supplementary 2. Movie S1: self-gelation process of MAGNCs@Gel.

Supplementary 3. Movie S2: demonstration of injectable properties of MAG-NCs@Gel.

\section{References}

[1] E. Masliah, E. Rockenstein, I. Veinbergs et al., "Dopaminergic loss and inclusion body formation in $\alpha$-Synuclein mice: implications for neurodegenerative disorders," Science, vol. 287, no. 5456, pp. 1265-1269, 2000.

[2] W. Poewe, K. Seppi, C. M. Tanner et al., "Parkinson disease," Nature Reviews. Disease Primers, vol. 3, no. 1, p. 21, 2017.

[3] D. Zhao, P. J. Feng, J. H. Liu et al., "Electromagnetized-nanoparticle-modulated neural plasticity and recovery of degenerative dopaminergic neurons in the mid-brain," Advanced Materials, vol. 32, no. 43, article 2003800, 2020.

[4] J. Liu, C. Liu, J. Zhang et al., "A self-assembled $\alpha$-Synuclein nanoscavenger for Parkinson's disease," ACS Nano, vol. 14, no. 2, pp. 1533-1549, 2020.

[5] K. J. Kuder, M. Zaluski, J. Schabikowski et al., "Novel, dual target-directed annelated xanthine derivatives acting on 
adenosine receptors and monoamine oxidase B," ChemMedChem, vol. 15, no. 9, pp. 772-786, 2020.

[6] M. Bologna, G. Paparella, A. Fasano, M. Hallett, and A. Berardelli, "Evolving concepts on bradykinesia," Brain, vol. 143, no. 3, pp. 727-750, 2020.

[7] Z. M. Chen, F. Y. Liu, Y. K. Chen et al., "Targeted delivery of CRISPR/Cas9-mediated cancer gene therapy via liposometemplated hydrogel nanoparticles," Advanced Functional Materials, vol. 27, no. 46, article 1703036, 2017.

[8] S. Xiong, Z. Li, Y. Liu et al., "Brain-targeted delivery shuttled by black phosphorus nanostructure to treat Parkinson's disease," Biomaterials, vol. 260, article 120339, 2020.

[9] R. Pandit, L. Y. Chen, and J. Götz, "The blood-brain barrier: physiology and strategies for drug delivery," Advanced Drug Delivery Reviews, vol. 165-166, pp. 1-14, 2020.

[10] J. Garcia-Pardo, F. Novio, F. Nador et al., "Bioinspired theranostic coordination polymer nanoparticles for intranasal dopamine replacement in Parkinson's disease," ACS Nano, vol. 15, no. 5, pp. 8592-8609, 2021.

[11] H. Zhou, L. Niu, L. Meng et al., "Noninvasive ultrasound deep brain stimulation for the treatment of Parkinson's disease model mouse," Research, vol. 2019, article 1748489, 2019.

[12] C. Y. Wu, B. Y. Li, Y. Zhang et al., "Intranasal delivery of paeoniflorin nanocrystals for brain targeting," Asian Journal of Pharmaceutical Sciences, vol. 15, no. 3, pp. 326-335, 2020.

[13] S. C. Li, X. Sun, L. Bi, Y. J. Tong, and X. Liu, "Research Progress on Natural Product Ingredients' Therapeutic Effects on Parkinson's Disease by Regulating Autophagy," Evidence-Based Complementary and Alternative Medicine, vol. 2021, Article ID 5538200, 11 pages, 2021.

[14] E. R. de Oliveira Junior, E. Truzzi, L. Ferraro et al., "Nasal administration of nanoencapsulated geraniol/ursodeoxycholic acid conjugate: towards a new approach for the management of Parkinson's disease," Journal of Controlled Release, vol. 321, pp. 540-552, 2020.

[15] M. Agrawal, S. Saraf, S. Saraf et al., "Stimuli-responsive In situ gelling system for nose-to-brain drug delivery," Journal of Controlled Release, vol. 327, pp. 235-265, 2020.

[16] C. Zheng, M. Li, and J. Ding, "Challenges and opportunities of nanomedicines in clinical translation," BIO Integration, vol. 2, no. 2, pp. 57-60, 2021.

[17] E. Ahmad, Y. H. Feng, J. P. Qi et al., "Evidence of nose-to-brain delivery of nanoemulsions: cargoes but not vehicles," Nanoscale, vol. 9, no. 3, pp. 1174-1183, 2017.

[18] J. Wang, P. Y. Li, Y. Yu et al., "Pulmonary surfactantbiomimetic nanoparticles potentiate heterosubtypic influenza immunity," Science, vol. 367, no. 6480, article aau0810, 2020.

[19] Z. K. Zhang, C. R. Liu, C. Li, W. Wu, and X. Q. Jiang, "Shape effects of cylindrical versus spherical unimolecular polymer nanomaterials on in vitro and in vivo behaviors," Research, vol. 2019, article 2391486, 2019.

[20] L. Wei, J. Chen, and J. J. N. Ding, "Sequentially stimuliresponsive anticancer nanomedicines," Nanomedicine, vol. 16, no. 4, pp. 261-264, 2021.

[21] S. Kobari, T. Kusakabe, M. Momota et al., "IL-33 is essential for adjuvant effect of hydroxypropyl- $\beta$-cyclodexrin on the protective intranasal influenza vaccination," Frontiers in Immunology, vol. 11, article 00360, 2020.

[22] T. A. Kulkarni, A. N. Bade, B. Sillman et al., "A year-long extended release nanoformulated cabotegravir prodrug," Nature Materials, vol. 19, no. 8, pp. 910-920, 2020.
[23] T. K. Chen, W. Liu, S. Xiong et al., "Nanoparticles mediating the sustained puerarin release facilitate improved brain delivery to treat Parkinson's disease," ACS Applied Materials \& Interfaces, vol. 11, no. 48, pp. 45276-45289, 2019.

[24] H. Guo, F. Li, H. Qiu, W. Xu, and X. J. R. Chen, "Synergistically enhanced mucoadhesive and penetrable polypeptide nanogel for efficient drug delivery to orthotopic bladder cancer," Research, vol. 2020, article 8970135, 2020.

[25] Y. Xiao, J. Chen, C. Wang, J. Ding, and W. Tao, "Editorial: Emerging micro- and nanotechnologies for medical and pharmacological applications," Frontiers in Pharmacology, vol. 12, article 648749, 2021.

[26] M. A. Oshi, J. Lee, M. Naeem et al., "Curcumin nanocrystal/pH-responsive polyelectrolyte multilayer core-shell nanoparticles for inflammation-targeted alleviation of ulcerative colitis," Biomacromolecules, vol. 21, no. 9, pp. 3571-3581, 2020.

[27] T. Domenech and P. S. Doyle, "High loading capacity nanoencapsulation and release of hydrophobic drug nanocrystals from microgel particles," Chemistry of Materials, vol. 32, no. 1, pp. 498-509, 2020.

[28] N. Gautam, J. M. McMillan, D. Kumar et al., "Lipophilic nanocrystal prodrug-release defines the extended pharmacokinetic profiles of a year-long cabotegravir," Nature Communications, vol. 12, no. 1, pp. 3453-3453, 2021.

[29] P. D. Godfrin, H. Lee, J. H. Lee, and P. S. Doyle, "Photopolymerized micelle-laden hydrogels can simultaneously form and encapsulate nanocrystals to improve drug substance solubility and expedite drug product design," Small, vol. 15, no. 6, article 1803372, 2019.

[30] J. Zhou, J. Li, X. du, and B. Xu, "Supramolecular biofunctional materials," Biomaterials, vol. 129, pp. 1-27, 2017.

[31] H. J. He, X. Y. Lin, J. Q. Guo, J. Q. Wang, and B. Xu, "Perimitochondrial enzymatic self-assembly for selective targeting the mitochondria of cancer cells," ACS Nano, vol. 14, no. 6, pp. 6947-6955, 2020.

[32] W. Wang, B. Y. Tan, J. R. Chen et al., "An injectable conductive hydrogel encapsulating plasmid DNA-eNOs and ADSCs for treating myocardial infarction," Biomaterials, vol. 160, pp. 69-81, 2018.

[33] X. Y. Lin, B. R. Xu, H. Zhu, J. R. Liu, A. Solovev, and Y. F. Mei, "Requirement and development of hydrogel micromotors towards biomedical applications," Research, vol. 2020, article 7659749, 2020.

[34] H. Madry, L. Gao, A. Rey-Rico et al., "Thermosensitive hydrogel based on PEO-PPO-PEO poloxamers for a controlled in situ release of recombinant adeno-associated viral vectors for effective gene therapy of cartilage defects," Advanced Materials, vol. 32, no. 2, article 1906508, 2020.

[35] H. Kawaguchi, "Functional polymer microspheres," Progress in Polymer Science, vol. 25, no. 8, pp. 1171-1210, 2000.

[36] X. Song, Z. X. Zhang, J. L. Zhu et al., "Thermoresponsive hydrogel induced by dual supramolecular assemblies and its controlled release property for enhanced anticancer drug delivery," Biomacromolecules, vol. 21, no. 4, pp. 1516-1527, 2020.

[37] S. J. Ge, J. J. Li, J. Geng, S. N. Liu, H. Xu, and Z. Z. Gu, “Adjustable dual temperature-sensitive hydrogel based on a selfassembly cross-linking strategy with highly stretchable and healable properties," Materials Horizons, vol. 8, no. 4, pp. 1189-1198, 2021. 
[38] S. J. P. McInnes, E. J. Szili, S. A. al-Bataineh et al., "Fabrication and characterization of a porous silicon drug delivery system with an initiated chemical vapor deposition temperatureresponsive coating," Langmuir, vol. 32, no. 1, pp. 301-308, 2016.

[39] N. Singh and L. A. Lyon, "Au nanoparticle templated synthesis of pNIPAm nanogels," Chemistry of Materials, vol. 19, no. 4, pp. 719-726, 2007.

[40] K. Akiyoshi, E. C. Kang, S. Kurumada, J. Sunamoto, T. Principi, and F. M. Winnik, "Controlled association of amphiphilic polymers in water: thermosensitive nanoparticles formed by self-assembly of hydrophobically modified pullulans and poly(N-isopropylacrylamides)," Macromolecules, vol. 33, no. 9, pp. 3244-3249, 2000.

[41] X. B. Gong, C. Y. Hou, Q. H. Zhang, Y. G. Li, and H. Z. Wang, "Thermochromic hydrogel-functionalized textiles for synchronous visual monitoring of On-DemandIn VitroDrug release," ACS Applied Materials \& Interfaces, vol. 12, no. 46, pp. 51225-51235, 2020.

[42] E. Liarou, S. Varlas, D. Skoulas et al., "Smart polymersomes and hydrogels from polypeptide-based polymer systems through alpha-amino acid N-carboxyanhydride ring-opening polymerization," Progress in Polymer Science, vol. 83, pp. 28$78,2018$.

[43] S. Xiong, J. S. Luo, Q. Wang et al., "Targeted graphene oxide for drug delivery as a therapeutic nanoplatform against Parkinson's disease," Biomaterials Science, vol. 9, no. 5, pp. 1705-1715, 2021.

[44] Y. Tan, D. Wang, H. Xu et al., "A fast, reversible, and robust gradient nanocomposite hydrogel actuator with waterpromoted thermal response," Macromolecular Rapid Communications, vol. 39, no. 8, 2018.

[45] H. H. Chen, P. C. Chang, S. P. Wey, P. M. Chen, C. Chen, and M. H. Chan, "Therapeutic effects of honokiol on motor impairment in hemiparkinsonian mice are associated with reversing neurodegeneration and targeting $\operatorname{PPAR} \gamma$ regulation," Biomedicine \& Pharmacotherapy, vol. 108, pp. 254$262,2018$.

[46] C. C. Weng, Z. A. Chen, K. T. Chao et al., "Quantitative analysis of the therapeutic effect of magnolol on MPTP-induced mouse model of Parkinson's disease using in vivo 18F-9-fluoropropyl-(+)-dihydrotetrabenazine PET imaging," PLoS One, vol. 12, no. 3, article 0173503, 2017.

[47] Y. Tan, D. Wang, H. Xu et al., "Rapid recovery hydrogel actuators in air with bionic large-ranged gradient structure," ACS Applied Materials \& Interfaces, vol. 10, no. 46, pp. 4012540131, 2018.

[48] J. Liu, G. Chen, M. Guo, and M. Jiang, "Dual stimuliresponsive supramolecular hydrogel based on hybrid inclusion complex (HIC)," Macromolecules, vol. 43, no. 19, pp. 80868093, 2010.

[49] J. G. Zhang, H. Yang, B. E. Abali, M. J. Li, Y. Xia, and R. Haag, "Dynamic mechanics-modulated hydrogels to regulate the differentiation of stem-cell spheroids in soft microniches and modeling of the nonlinear behavior," Small, vol. 15, no. 30, article 1901920, 2019.

[50] P. Chakraborty, Y. M. Tang, T. Yamamoto et al., "Unusual two-step assembly of a minimalistic dipeptide-based functional hypergelator," Advanced Materials, vol. 32, no. 9, article 1906043, 2020.

[51] Y. H. Wu, H. B. Wang, F. Gao, Z. Y. Xu, F. Y. Dai, and W. G. Liu, "An injectable supramolecular polymer nanocomposite hydrogel for prevention of breast cancer recurrence with theranostic and mammoplastic functions," Advanced Functional Materials, vol. 28, no. 21, p. 1801000, 2018.

[52] J. Zheng, R. Fan, H. Wu et al., "Directed self-assembly of herbal small molecules into sustained release hydrogels for treating neural inflammation," Nature Communications, vol. 11, p. 1640, 2020.

[53] J. E. Mealy, J. J. Chung, H. H. Jeong et al., "Injectable granular hydrogels with multifunctional properties for biomedical applications," Advanced Materials, vol. 30, no. 20, article $1705912,2018$.

[54] H. Wang, Y. Wu, C. Cui, J. Yang, and W. Liu, "Antifouling super water absorbent supramolecular polymer hydrogel as an artificial vitreous body," Advancement of Science, vol. 5, no. 11, article 1800711, 2018.

[55] C. Loebel, C. B. Rodell, M. H. Chen, and J. A. Burdick, "Shearthinning and self-healing hydrogels as injectable therapeutics and for 3D-printing," Nature Protocols, vol. 12, no. 8, pp. 1521-1541, 2017.

[56] di Stefano, L. Marinelli, P. Eusepi et al., "Synthesis and biological evaluation of novel selenyl and sulfur-l-dopa derivatives as potential anti-Parkinson's disease agents," Biomolecules, vol. 9, no. 6, p. 239, 2019.

[57] C. Rosenblad, Q. Li, E. Y. Pioli et al., "Vector-mediated L-3,4dihydroxyphenylalanine delivery reverses motor impairments in a primate model of Parkinson's disease," Brain, vol. 142, no. 8, pp. 2402-2416, 2019. 